\title{
REVIEW
}

\section{Infant and toddler diabetes}

\section{Declan Cody}

Arch Dis Child 2007;92:716-719. doi: 10.1136/adc.2006.112896

$\mathrm{T}$ he incidence of type 1 diabetes mellitus continues to increase with an estimated rise in incidence rates of between $3 \%$ and $5 \%$ per year. ${ }^{12}$ This increase has been most pronounced in the pre-school age group. ${ }^{3}$

The rising incidence has been paralleled by the increasing intensity of management of this condition. The Diabetes Control and Complications Trial (DCCT) study group demonstrated the importance of intensive diabetes management and subsequent strict glycaemic control, with significant reductions in microvascular complications with small changes in HbAlc. ${ }^{5}$ However, intensive diabetes management is associated with a potential increased risk of severe hypoglycaemia. ${ }^{67}$ Efforts to achieve near-normoglycaemia as early as possible after diagnosis should be considered based on the "tracking phenomenon" data which suggest a correlation between metabolic control in the early stages of treatment and that in subsequent years. ${ }^{8}$

The importance of intensive diabetes management has significant implications for toddlers and infants diagnosed with diabetes and for the children's diabetes services who look after them. This age group has particular features and clinical characteristics which makes their care distinct from that of older children with diabetes. This article will review the main clinical areas in the management of these children.

\section{EPIDEMIOLOGY}

Oxford data suggest an incidence rate in the UK of type 1 diabetes mellitus in children $<2$ years of age of about 1:15 000. ${ }^{4}$ Approximately $4 \%$ of children with type 1 diabetes are less than 2 years of age at diagnosis. ${ }^{9}$ This suggests that there are several hundred children being cared for in the UK and Ireland who were diagnosed at this age. These relatively small numbers must necessarily limit an individual diabetes clinic team's experience in the management of infants and toddlers with diabetes.

It is thus vital for diabetes health professionals in training to have experience and exposure to very young children with diabetes. The knowledge that diabetes is associated with a shortened lifespan of up to 15 years becomes even more significant for children diagnosed at such an early age. Furthermore, the belief in pre-pubertal protection from the microvascular complications of diabetes, and particularly for retinopathy, is now readily disputed. ${ }^{10-12}$

Recent surveys in both the UK and Ireland (unpublished) on services for children with diabetes have shown that some clinics are seeing relatively small $(<70)$ numbers of patients. ${ }^{13}$ In these circumstances, a "hub and spoke" approach to the management of toddlers, with joint care arrangements between regional and district general hospitals, should be encouraged, particularly in relation to the employment of newer treatment strategies such as insulin pump therapy and continuous glucose monitoring. The potential for an increased morbidity and mortality risk in this age group requires optimal levels of care to be provided to these children and their families from the outset.

\section{CLINICAL CHARACTERISTICS}

At initial presentation very young children have an increased risk of diabetic ketoacidosis and cerebral oedema compared to older children. Some centres have reported that between $53 \%$ and $85 \%$ of their patients diagnosed with diabetes at less than 2 years of age presented with diabetic ketoacidosis. ${ }^{14}$ Despite a more rapid and severe presentation they have a lower HbAlc at diagnosis and a shorter duration of symptoms. This finding of lower glycosylated haemoglobin suggests that the increased risk of diabetic ketoacidosis may be due to a more severe form of diabetes at presentation rather than a delay in picking up signs and symptoms of diabetes in this age group.

Komulainen's group found that very young children had higher titres of diabetes-related autoantibodies at diagnosis than older children, again indicating a more aggressive autoimmune insult. ${ }^{9}$ Evidence for a more potent form of disease and more rapid pancreatic $\beta$ cell loss at this age is also shown by the absence or reduced duration of the partial remission (honeymoon) phase along with the higher insulin requirements in the first 6 months after diagnosis. ${ }^{15}$

Beyond the post-diagnosis period, toddlers and infants with diabetes have certain characteristics which differentiate them from older children with diabetes. They are very variable with respect to daily activities and food intake. Furthermore, along with the practical problems of administering insulin to a squirming infant, this age group is also very sensitive to tiny doses of insulin and can have extremely variable blood sugar control with unpredictable fluctuations. ${ }^{16}$ Frequent illnesses and rapid growth spurts at this age can add to the difficulties with blood sugar control.

\section{FOOD AND TODDLERS WITH DIABETES}

Issues around food are often a source of frustration for parents of very young children with diabetes. Along with the difficulties associated with prolonged nocturnal fasting and regular bottle feeds are the additional problems of food refusal.

In general terms the normal food provided for infants (ie, breast or formula feed) is entirely satisfactory for infants with diabetes provided they are fed regularly. In some centres a grazing style approach is encouraged in later infancy to reduce 
the risk of hypoglycaemia. ${ }^{17}$ In our clinic we encourage a more regular meal pattern consisting of complex carbohydrate-based meals and snacks (eg, fruit, yoghurt, cereals) to reduce the risk of hypoglycaemia with continuous grazing discouraged to limit potential hyperglycaemia. In general, many toddlers will eat every couple of hours and blood sugar recordings may well reflect this fact with most sugar readings probably reflecting the post-prandial milieu.

Food refusal should generally be dealt with responsibly and similarly to toddlers without diabetes. Young children becoming increasingly independent can recognise parental stress and quickly learn to use their diabetes as a way of getting their favourite food. It is important to emphasise parental patience and to encourage parents not to use food bribes to get their children to eat and to encourage a healthy approach to eating involving all food groups. One suggestion is to offer only two food choices and if not eaten within 2030 min to remove the food without comment. New foods can be introduced, again without comment, alongside the foods that they like. A family-centred approach to eating time and avoidance of distractions are encouraged. If the issues of food refusal continue, a reduction in insulin may occasionally be required.

\section{FAMILY FUNCTIONING}

The diagnosis of type 1 diabetes, a difficult and stressful time for parents, may be exacerbated for parents of infants and toddlers. ${ }^{18}$

A greater burden may be felt by parents as these children are totally dependent on them for both their normal child welfare as well as regular decision making concerning medical management. Vigilance with respect to potential hypoglycaemia adds to the burden. A heightened sense of grief at diagnosis may be reported and particularly so if the child is critically ill. ${ }^{19}$ The necessity to have to administer frequent injections as well as regular blood tests is often contrary to a parent's natural instinct not to cause pain to their child. Major lifestyle changes may be required for the parents including change in employment. They can experience isolation and exhaustion particularly as family and friends may be fearful in helping with looking after the child.

In a qualitative analysis on the adjustment to diabetes mellitus in pre-school children and their mothers, the mothers felt that their children showed more "internalising" features (anxiety, withdrawal), while they themselves perceived greater family disruption and concern about hypoglycaemia than for parents of older children with diabetes. ${ }^{20}$

Hatton described three phases in terms of coping with diabetes in the very young including initial grief, guilt and anger at diagnosis, followed by the stage of caring for the child at home which is described as a time of survival and the final stage of adaptation as parents learn to trust others and build support systems. ${ }^{21}$

The lack of psychological support in helping vulnerable families, recently highlighted in the Services for Children with Diabetes surveys, remains of huge concern. ${ }^{13}$ This deficiency, along with the need for training of staff in nurseries and preschools to help with blood sugar testing and insulin management, is an area which needs to be urgently addressed.

\section{HYPOGLYCAEMIA}

Hypoglycaemia is a cause of great anxiety for all parents of children with diabetes and particularly for parents of infants and toddlers. Very young children are unable to communicate symptoms of hypoglycaemia to their parents, with pallor or change in behaviour being sometimes the only obvious sign. Asymptomatic nocturnal hypoglycaemia in this age group is a particular issue due to prolonged overnight fasting. One study involving continuous glucose monitoring over a 3 day period of a group of very young children with diabetes, revealed periods of nocturnal hypoglycaemia varying from 10 to $480 \mathrm{~min}$, with an average of almost an hour, in nine of the 11 patients studied..$^{22}$ Schwenk's retrospective review found that more than half of his cohort of children diagnosed with diabetes at less than 2 years of age had a severe hypoglycaemic seizure in the 2 years after diagnosis, compared with $13 \%$ of $5-9$ year olds. There was no identifiable cause for their seizure in $80 \%$ of the toddler group. ${ }^{23}$

The effect of severe hypoglycaemia on the developing brain of the infant and toddler in terms of cognitive impairment remains a controversial area. ${ }^{24}$ Rovet's prospective evaluation on an older population (mean age 12 years) found deficits in perceptual, motor and memory tasks in those with severe hypoglycaemic seizures. ${ }^{25}$ Further studies showed significant differences in neuropsychological profiles between diabetic and control children with the difference being attributed to hypoglycaemia. ${ }^{26}{ }^{27}$ Extrapolating these findings to a younger and more vulnerable brain in terms of cognitive impairment suggests that a more gentle and less aggressive approach to blood sugar control may be warranted. A recent position paper from the American Diabetes Association suggested that an Hbalc reading of 7.5-8.5 was acceptable and recommended for children under 6 years of age. ${ }^{28}$

However with the support of a specialised multi-disciplinary team, including a $24 \mathrm{~h}$ advice hotline, these young children may benefit from treatment intensification without the purported increase in severe hypoglycaemia. ${ }^{29}$ This increased access along with the use of the newer continuous glucose sensors to help with diagnosing asymptomatic or nocturnal hypoglycaemia should be considered for families of all infants and toddlers with diabetes. Hypoglycaemia, per se, should not be a reason to accept higher blood sugar recordings but should stimulate a more concerted effort for optimal control.

\section{INSULIN THERAPY}

In the UK and Ireland many children in the toddler age group are started on once daily insulin. Our particular practice is to administer insulin via a syringe, to allow free-mixing, initially once or twice daily. Due to difficulties with unpredictable blood sugar control at this age, there is an increasing tendency to use a multiple-injection regime or insulin pump therapy. Surely it is now time to question the use of the non-physiological single injection of long-acting insulin to treat diabetes at this age. At present, however, there is no evidence from randomised controlled trials for this age group to compare the use of once-daily long-acting insulin versus multiple-injection therapy in terms of clinical and metabolic outcomes to inform practice. Collaborative studies should be encouraged to examine this question.

Despite the fact that the newer long-acting insulin analogues are unlicensed for children under 6 years of age, there is increasing anecdotal and published evidence of their efficacy in this age group, particularly in relation to reduced hypoglycaemia risk. ${ }^{30} 31$ A more targeted therapeutic and physiological approach to diabetes management with the newer insulin analogues, incorporating carbohydrate-counting and insulinto-carbohydrate ratios, should be considered for each individual case. For those toddlers already on rapid-acting insulin analogues at mealtimes, it may be more sensible, and safer, to administer the insulin after the meal to match the insulin to the food eaten. This might facilitate increased flexibility and help to avoid the struggles that might otherwise ensue. ${ }^{32}$ The importance of specialised paediatric diabetes dieticians with experience in looking after toddlers with diabetes, and closely 
involved with their overall management, cannot be overstressed.

\section{Insulin pump therapy}

Recent advances in insulin pump therapy have remarked on its safety profile at all ages. The pertinent question to be addressed is whether the youngest children may actually benefit more from insulin pump therapy than any other age group. ${ }^{33}$ This reasoning is based on the previously stated difficulties including their highly variable food intake and activity levels along with their tendency to unpredictable hypoglycaemia. Insulin pump therapy potentially allows greater control with respect to altering basal rates, with insulin infusion even being stopped for a time and tiny boluses administered at mealtimes. It may increase family flexibility and reduces the need for multiple injections.

While there is some retrospective evidence to suggest that insulin pump therapy in this age group results in improved glycaemic control with no increase in adverse effects, ${ }^{34}{ }^{35}$ more recent prospective randomised studies did not find any significant improvements in glycaemic control compared to multiple-injection therapy. ${ }^{36}{ }^{37}$

The recommendation for starting insulin pump therapy at this age may therefore be based more on quality-of-life issues with potential huge reductions in parental stress rather than on measurable clinical parameters. Patient selection, bearing in mind that pump therapy may not suit all patients and families, is an essential factor in ensuring an effective insulin pump service delivery. ${ }^{38}$ While National Institute for Clinical Excellence (NICE) criteria do not explicitly include quality-oflife measures for children to qualify for insulin pump therapy the clinician should view the needs of this age group as distinct from other age groups and address them as such. ${ }^{39}$

\section{ADVANCES}

Recent genetic advances involving infants diagnosed with permanent neonatal diabetes at less than 6 months of age have identified mutations affecting the $\mathrm{K}_{\text {ATP }}$ channel of the pancreatic $\beta$ cell in over $40 \%$ of this group. ${ }^{40}{ }^{41}$ The presence of activating mutations in the Kir 6.2 subunit of this channel allows the therapeutic possibility of discontinuing insulin therapy and switching to oral sulphonylurea therapy. All infants diagnosed with diabetes at less than 6 months of age should thus have genetic testing to check for this mutation. The major aspects of permanent neonatal diabetes are beyond the scope of this article.

\section{CONCLUSION}

The rising incidence of type 1 diabetes, particularly in the very young child, has huge implications for both parents and health professionals. Infants and toddlers have various age-related characteristics making the management of their diabetes distinct from that of older children. These clinical and psychosocial issues often mean that both parents and health professionals struggle with ensuring good clinical control while minimising family stresses. Providing more physiological insulin regimes should be strongly encouraged. The importance of a specialised multi-disciplinary team in providing on-going education and close support to families of very young children with diabetes is an essential component of an effective children's diabetes service. It is essential that diabetes team members are experienced in managing diabetes in this age group and this perhaps should be a prerequisite for consultant and paediatric diabetes specialist nursing appointments. Close cooperation between regional and district general hospitals in looking after this particular age group should be encouraged.

Competing interests: None declared.

\section{REFERENCES}

1 Gillespie KM, Bain SC, Barnett AH, et al. The rising incidence of childhood type 1 diabetes and reduced contribution of high-risk haplotypes. Lancet 2004;364:1699-1700.

2 Rasgasamai JJ, Greenwood DC, McSporran B, et al. Rising incidence of type 1 diabetes in Scottish children, 1984-93. The Scottish Study Group for the Care of Young Diabetics. Arch Dis Child 1997;77(3):210-13.

3 Dahlquist G, Hustonen L. Analysis of a 15 year prospective incidence study of childhood diabetes onset: time trends and climatological factors. Int J Epidemiol 1994;23:1234-41.

4 Gardner SG, Bingley PJ, Satwell PA, et al. Rising incidence of insulin dependent diabetes in children aged under 5 years in the Oxford region: time trend analysis. The Barts-Oxford Study Group. BMJ 1997;315:713-7.

5 The Diabetes Control and Complications Trial Research Group. Effects of intensive diabetes treatment on the development and progression of long-term complications in adolescents with insulin-dependent diabetes mellitus. N Engl J Med 1993;329:977-86.

6 The Diabetes Control and Complications Trial Research Group. Hypoglycemia in the Diabetes Control and Complications Trial. Diabetes 1997;46:271-86.

7 Nordfeldt S, Ludvigsson J. Adverse events in intensively treated children and adolescents with type 1 diabetes. Acta Paediatr 1999;88:1184-93.

8 Swift PGF. Insulin treatment at onset of diabetes. Horm Res 2002;57(1):93-6.

9 Komulainen J, Kulmala P, Savola K. Clinical, autoimmune and genetic characteristics of very young children with type 1 diabetes. Diabetes Care 1999;22:1950-5.

10 Svensson M, Eriksson JW, Dahlquist G. Early glycemic control, age at onset and development of microvascular complications in childhood-onset type 1 diabetes. Diabetes Care 2004;27(4):955-62.

11 Donaghue KC, Fung AT, Hing S, et al. The effect of prepubertal diabetes duration on diabetes. Microvascular complications in early and late adolescence. Diabetes Care 1997;20:77-80.

12 Holl RW, Lang GE, Grabert M, et al. Diabetic retinopathy in pediatric patients with type-1 diabetes: effect of diabetes duration, prepubertal and pubertal onset of diabetes, and metabolic control. Pediatrics 1998;132:790-4.

13 Edge JA, Swift PGF, Anderson W, et al. Diabetes services in the UK: fourth national survey: are we meeting NSF standards and NICE guidelines? Arch Dis Child 2005;90:1005-9.

14 Paul P, Ghatak A, Kerr S, et al. Severe metabolic decompensation at presentation of diabetes mellitus in children aged $<2$ years. Arch Dis Child 2005;90:A19-22.

15 Muhammad BJ, Swift PGF, Raymond NT, et al. Partial remission phase of diabetes in children younger than age 10 years. Arch Dis Child 1999;80:367-9.

16 Raine JE, Donaldson MDC, Gregory JW, et al. Practical endocrinology and diabetes in children, 2nd edn. Oxford: Blackwell, 2006:1-33.

17 Diabetes Australia. Parents \& carers. Information for parents of kids aged 0-2 years. http://www.diabeteskidsandteens.com.au (accessed 8 March 2007).

18 Kushion W, Salisbury PJ, Seitz KW. Issues in the care of infants and toddlers with insulin dependent diabetes mellitus. Diabetes Educ 1991;17(2):107-10.

19 Anderson BJ. Children with diabetes mellitus and family functioning: translating research into practice. J Pediatr Endocrinol Med 2001;14:645-52.

20 Wysocki T, Huxtable K, Linscheid TR. Adjustment to diabetes mellitus in preschoolers and their mothers. Diabetes Care 1989;12(8):524-9.

21 Hatton DL, Canam CC, Thorne S, et al. Parents perceptions of caring for an infant or toddler with diabetes. J Adv Nurs 1995;22:569-77.

22 Deiss D, Kordonouri K, Meyer K, et al. Long hypoglycaemic periods detected by subcutaneous continuous glucose monitoring in toddlers and pre-school children with diabetes mellitus. Diabetic Med 2001;18:333-8.

23 Lteif AN, Schwenk WF. Type 1 diabetes mellitus in early childhood: glycemic control and associated risk of hypoglycaemic reactions. Mayo Clin Proc 1999;74:211-16.

24 Ryan C, Vega A, Drash A. Cognitive deficits in adolescents who developed diabetes in early life. Pediatrics 1985;75:921-7.

25 Rovet JF, Ehrlich RM. The effect of hypoglycaemic seizures on cognitive function in children with diabetes: a seven year prospective study. J Pediatr $1999 ; 134: 503-6$

26 Hershey T, Bhargava, N, Sadler M, et al. Conventional versus intensive diabetes therapy in children with type 1 diabetes: effects on memory and motor speed. Diabetes Care 1999;22(8):1318-24.

27 Northam EA, Anderson PJ, Jacobs R, et al. Neuropsychological profiles of children with type 1 diabetes 6 years after disease onset. Diabetes Care $2001 ; 24(9): 1541-7$.

28 Silverstein J, Klingensmith K, Copeland L. Care of children and adolescents with type 1 diabetes: a statement of the American Diabetes Association. Diabetes Care 2005;28(1):186-212.

29 Kiess W, Kapellen T, Siebler T, et al. Practical aspects of managing preschool children with type 1 diabetes. Acta Paediatr 1998;425:67-71.

30 Hathout EH, Fujishige L, Geach J, et al. Effect of therapy with insulin glargine (lantus) on glycemic control in toddlers, children and adolescents with diabetes. Diabetes Technol Ther 2003;5(5):801-6.

31 Dixon B, Chase HA, Burdick J, et al. Use of insulin glargine in children under age 6 with type 1 diabetes. Pediatr Diabetes 2005;6(3):150-4.

32 Rutledge KS, Chase HP, Klingensmith GJ, et al. Effectiveness of postprandial Humalog in toddlers with diabetes. Pediatrics 1997; 100(6):968-72.

33 Winzimer SA, Swan KL, Sikes KA, et al. Emerging evidence for the use of insulin pump therapy in infants, toddlers and pre-school children with type 1 diabetes. Pediatr Diabetes 2006;7(4):15-19. 
34 Ahern JH, Boland EA, Doane R, et al. Insulin pump therapy in pediatrics: a therapeutic alternative to safely lower $\mathrm{HbAlc}$ levels across all age groups. Pediatr Diabetes 2002;3:10-15.

35 Mack-Fogg JE, Orlowski CC, Jospe N. Continuous subcutaneous insulin infusion in toddlers and children with type 1 diabetes is safe and effective. Pediatr Diabetes 2005:6(1):17-21.

36 Fox LA, Buckloh LM, Smith SD, et al. A randomized controlled trial of insulin pump therapy in young children with type 1 diabetes. Diabetes Care 2005;28(6): 1277-81.

37 DiMeglio LA, Pottorff TM, Boyd SR, et al. A randomized controlled study of insulin pump therapy in diabetic preschoolers. J Pediatr 2004;145:380-4.
38 Eugster EA, Francis G. Position statement: continuous subcutaneous insulin infusion in very young children with type 1 diabetes. Pediatrics 2006;118(4): 1244-9.

39 National Institute for Clinical Excellence (NICE). Guidance on the use of continuous subcutaneous insulin infusion for diabetes, Technology appraisal guidance no 57. London, UK: NICE, 2003.

40 Pearson ER, Fletchner I, Niolstad PR, et al. Switching from insulin to oral sulfonylureas in patients with diabetes due to Kir6.2 mutations. N Engl J Med 2006;355(5):467-76.

41 Hattersley AT, Ashcroft FM. Activating mutations in Kir6.2 and neonatal diabetes. Diabetes 2005;54:2503-15.

\section{IMAGES IN PAEDIATRICS}

\section{Meningococcal septicaemia and dental changes}

A case is described where localised tissue necrosis in meningococcal septicaemia led to profound dental disturbances in later life. Neisseria meningitides infection may lead to disseminated intravascular coagulation (DIC), necrosis and infarction of organs, haemorrhage into the internal organs, ${ }^{1-3}$ hypocalcaemia ${ }^{2}$ and post-recovery bone abnormalities. ${ }^{3}$ Dental complications have been reported with disruption of odontogenesis or delayed tooth eruption. ${ }^{134}$

\section{Case report}

An 8-year-old girl was referred because of the discolouration of her permanent teeth.

She had had an episode of meningococcal septicaemia at 13 months of age. The main findings at that time were DIC with necrosis of part of the facial skin of her upper lip and fasciitis affecting her limbs.

Extra-oral examination at 8 years of age revealed old scars above the upper lip. Intra-oral examination showed that two lower permanent and one primary upper incisor teeth were severely hypoplastic.

Intra-oral radiographs showed a complicated image in the area of the upper front teeth (fig 1). There was a disturbance in the formation of the permanent upper lateral incisors and the upper left permanent canine. The crown of the upper left permanent central incisor (21) had stopped developing after the formation of the incisal edge. The other upper central incisor (11) presented with a similar image but tooth development seemed to have recommenced, probably with resolution of the septicaemia.

\section{Discussion}

The teeth do not all form at the same time and permanent incisors have usually initiated at or about the time of birth.
Tooth structure does not remodel and so changes in the form of teeth do not "heal" but go on to form a permanent record. Disturbances in the permanent dentition usually become obvious at 6-7 years of age when eruption of the permanent teeth begins.

It is difficult to discern the level at which the meningitic septicaemia affected the process of tooth development. Walton et al suggested that the occurrence of enamel defects was due to a probable subclinical premaxillary osteomyelitis. ${ }^{3}$

The purpose of this article is to inform paediatrician colleagues about such possible dental complications and to suggest that they might guide the families affected to seek dental care offered by specialist paediatric dentists.

\section{Siapka, P J M Crawford Child Dental Health, Dental School, Bristol, UK}

Correspondence to: P J M Crawford, Child Dental Health, Dental School, Bristol BS1 2LY, UK; peter.crawford@bristol.ac.uk

Competing interests: None.

\section{References}

1 Coyne BMC, Montague T. Teeth grinding, tongue and lip biting in a 20-monthold boy with meningococcal septicaemia. Report of a case. Int J Paediatr Dent 2002; 12:277-80.

2 Baines PB, Hart CA. Severe meningococcal disease in childhood. Br J Anaesth 2003;90(1):72-83.

3 Walton AG, Meechan JG, Welbury RR. Meningococcal septicemia and disseminated intravascular coagulation affecting the premaxillary permanent tooth germs. J Dent Child 1998;65(3):191-3.

4 Faibis S, Widmer R, Sapir S. Meningococcal septicaemia and dental complications: a literature review and two cases. Int J Paediatr Dent 2005; 15:213-19.
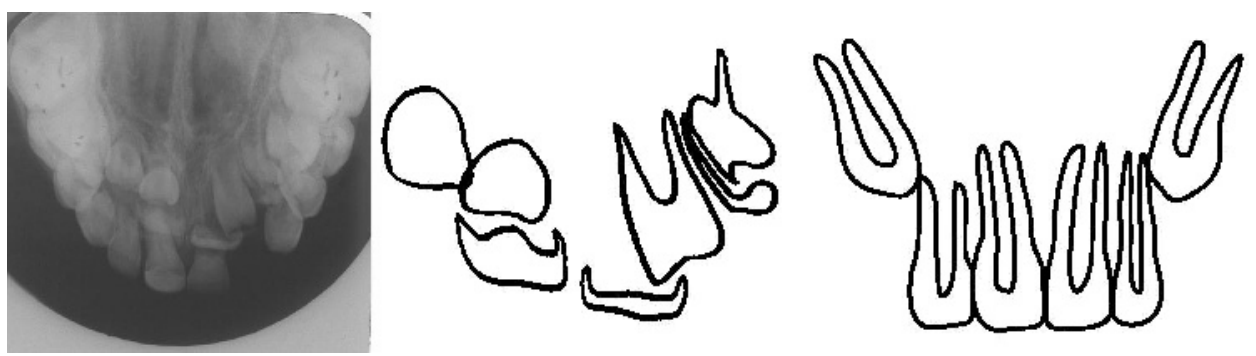

Figure 1 Left: upper occlusal radiograph. Middle: line drawing showing the identified tooth fragments Right: line drawing showing expected tooth development at this age. 